\title{
Introduction to Smart Mobility Ecosystems and Services Minitrack
}

\author{
Juho Lindman \\ University of Gothenburg \\ juho.lindman@ait.gu.se
}

\author{
Matti Rossi \\ Aalto University School of Business \\ matti.rossi@aalto.fi
}

\author{
Virpi Kristiina Tuunainen \\ Aalto University School of Business \\ virpi.tuunainen@aalto.fi
}

This new minitrack is the first HICSS minitrack focusing on different aspects of Smart Mobility Ecosystems and Services. Transportation is changing and this change is driven by technology-driven trends: autonomy, connectivity, electrification and diverse mobility business models of shared vehicles. At the same time there is a drive towards more sustainable transportation. Digital platforms and services are essential for orchestrating sustainable smart mobility service ecosystems: data that represents accurately for instance location of passengers and service providers, weather, usage and maintenance is a cornerstone for service design. Identification and communication systems that link specific physical things to specific digital addresses offer possibilities to communicate, transact, pay, build trust, sense and activate "things" from the internet - and design novel services based on this generated data. Autonomous vehicles, electric cars and mobility related sharing economy services are all built on platform thinking, as are many services reducing the need for travel to begin with.

In addition to usage of mobility value services, we are also interested in their design and development, as well as related service innovations. Furthermore, social, societal and potential customer segmentation issues are of great interest.

The three papers in this minitrack deal with services in this area from different perspectives.

In the first paper, "Trust is Good, Control is Better - Customer Preferences Regarding Control in Teleoperated and Autonomous Taxis", Katharina Keller (Goethe University Frankfurt), Christian Zimmermann (Robert Bosch GmbH), Jan Zibuschka (Robert Bosch GmbH), and Oliver Hinz (Goethe University Frankfurt), analyze the preferences of potential users regarding teleoperable robotaxis. Within the framework of a cluster analysis, the authors look at the results in segments and analyze possible moderating effects with variance analysis.

In the second paper, "A Probabilistic Location Prediction Approach to Optimize Dispatch Processes in the Ride-Hailing Industry", Keven Richly, Rainer Schlosser, Janos Brauer, and Hasso Plattner (all from Hasso-Plattner-Institut, Potsdam), present an approach to predict probability distributions for ride-hailing provider drivers' future locations. These are calculated based on patterns observed in past trajectories and the applicability and accuracy of the proposed algorithm is evaluated on a large real-world trajectory dataset of a transportation network company.

In the third paper, "How the Discourse of Urban Smart Mobility Portrays the Role of Automobility after 'The End of Car Ownership'", Arto Lanamäki (University of Vaasa), examines TeoCO ('The End of Car Ownership') as an agenda framing for policy and marketing purposes. The slogan establishes private car use - and the negative externalities of automobility - as the baseline comparison for new digital mobility services. This discourse is then situated within the theory of urban fabrics, to argue how cities need less automobility.

Together, these three papers present a snapshot of the emerging issues relevant to the field. Furthermore, this minitrack is intended to provide a venue to present findings, create future research collaborations around the emerging phenomenon and initiate debate on the future of these services. 